\title{
Método Baseado em Morfologia Matemática e BM3D para Redução de Ruído em Imagens Dentais de TC de Baixa Radiação
}

\author{
Rômulo Marconato Stringhini ${ }^{1}$, Daniel Welfer ${ }^{1}$ \\ ${ }^{1}$ Departamento de Computação Aplicada \\ Universidade Federal de Santa Maria (UFSM) - Santa Maria, RS - Brasil \\ rstringhini@inf.ufsm.br, welfer@gmail.com
}

\begin{abstract}
The impact in reducing the radiation dose in computed tomography (CT) exams is directly related to the quality of the images obtained in this exam. Such images are degraded by undesirable artifacts, known as noise. In order to improve the quality of these images and provide an accurate medical diagnosis, it is necessary to apply noise reduction techniques. In this article, it is proposed a method to filter noise in low-dose dental CT images, based on mathematical morphology and block-matching 3D (BM3D) filtering. Experimental results of the proposed method were compared with several existing methods and validated using the PSNR, SSIM and MSE metrics. Through several experiments, the proposed method demonstrated superior performance compared to the analyzed filters, reducing noise and preserving details in a more satisfactory way.
\end{abstract}

Resumo. O impacto em reduzir a dose de radiação em exames de tomografia computadorizada (TC) está diretamente relacionado à qualidade das imagens. Tais imagens são degradadas por artefatos indesejáveis, conhecidos como ruído. Diante disso, para melhorar a qualidade destas imagens e fornecer um diagnóstico médico preciso, é necessário aplicar técnicas que sejam capazes de reduzir ruído. Neste artigo, é proposto um método para filtrar ruído em imagens dentais de TC de baixa radiação, utilizando operadores da morfologia matemática e filtragem Block-Matching 3D (BM3D). Os resultados do método proposto foram comparados com diversos filtros existentes e validados utilizando as métricas PSNR, SSIM e MSE. Através de diversos experimentos, o método proposto demonstrou performance superior aos filtros analisados, reduzindo ruído e preservando detalhes de forma mais satisfatória.

\section{Introdução}

No campo de imagens médicas, a tomografia computadorizada (TC) é uma ferramenta de extrema importância para examinar as estruturas internas e diagnóstico médico preciso. Nesse exame, aumentando a dose de radiação implicará em imagens de melhor qualidade. Contudo, isso é prejudicial à saúde do paciente. Diante disso, a fim de preservar a saúde, dimimnuir o risco de doenças que altas doses de radiação podem acarretar, a comunidade médica têm se dedicado em diminuir essa dosagem em exames de tomografia computadorizada. Atualmente, é seguido o princípio ALARA (As Low As Reasonably Achievable), que tem como objetivo minimizar a dose de radiação em um nível "tão baixo quanto razoavelmente possível”, mantendo uma precisão diagnóstica aceitável [Li et al. 2014]. 
Imagens adquiridas em exames de TC de baixa radiação são, geralmente, degradadas por ruído e tem sua qualidade prejudicada, podendo degradar estruturas importantes e afetar o diagnóstico médico [Diwakar and Kumar 2018].

Diversos filtros foram propostos ao longo dos anos com a finalidade de reduzir ruído e preservar a integridade de detalhes importantes de imagens. Uma técnica de filtragem de ruído amplamente utilizada é o filtro da mediana [Pitas and Venetsanopoulos 1992]. Esse filtro tenta restaurar o valor da intensidade do pixel corrompido calculando a mediana dos valores das intensidades dos pixels vizinhos [El Hassani and Majda 2016]. Entretanto, esse tipo de filtragem não apenas reduz o ruído presente como também danifica detalhes estruturais [Feng et al. 2014]. Outra técnica muito conhecida e utilizada é a filtragem Gaussiana [Bergholm 1987]. Quando utilizado, esse filtro é eficiente em termos de redução de ruído porém borra bordas, detalhes e também dá origem à bordas fantasmas [Bergholm 1987].

Proposto por [Tomasi and Manduchi 1998], o filtro bilateral suaviza o ruído, preservando as bordas das estruturas das imagens. Cada pixel é substituído pela média ponderada dos valores das intensidades de seus pixels vizinhos, levando em consideração sua proximidade e similaridade [Tomasi and Manduchi 1998]. O filtro bitônico, proposto por [Treece 2016], apresenta melhor preservação de borda e detalhes do que o filtro da mediana e maior capacidade de redução de ruído do que o filtro Gaussiano. Além desses exemplos, têm-se filtragem por transformada wavelet, introduzido por [Donoho and Johnstone 1995], filtro guiado [He et al. 2013], BM3D (Block-Matching 3D) [Dabov et al. 2007], NLM (Non-Local Means) [Buades et al. 2005], AWMF (Adaptive Weighted Median Filter [Loupas et al. 1989], entre outros.

Além disso, recentemente, filtros para reduzir ruídos baseados em operadores da morfologia matemática, como dilatação, erosão, abertura e fechamento, vem se tornando cada vez mais comum [Ze-Feng et al. 2007]. Ademais, tais operadores são eficientes para diversos tipos de finalidade na área processamento de imagens, seja para reduzir ruído, segmentar estruturas, extrair informações ou comprimir imagens.

Entretanto, há uma dificuldade considerável em encontrar, na literatura, métodos que visam tratar, especificamente, de ruído em imagens dentais de tomografia computadorizada de baixa radiação.

Diante disso, nesse artigo, é apresentado um método baseado em operadores da morfologia matemática e filtragem BM3D (Block-Matching 3D). O método proposto tem como objetivo reduzir ruído e preservar detalhes em imagens dentais de tomografia computadorizada de baixa radiação. O decorrer desse trabalho está disposto a seguir: na Seção 2 será apresentada uma breve fundamentação teórica; na Seção 3 é apresentado a metodologia proposta; resultados experimentais são mostrados na Seção 4; a Seção 5 contém uma análise detalhada dos resultados obtidos e, por fim, o trabalho é concluído na Seção 6.

\section{Fundamentação Teórica}

\subsection{Operadores Básicos da Morfologia Matemática}

A principal característica da morfologia matemática consiste em extrair informações relativas à geometria e topologia de uma imagem através de um elemento estruturante 
[Marques Filho and Neto 1999]. Os dois principais operadores utilizados desse campo são os operadores de dilatação e erosão.

O operador de dilatação $\delta$ sobre uma imagem $f$ por um elemento estruturante $B$ em um determinado pixel $x$ é o valor máximo de $f$ na janela definida por $S$ centrada em $x$. Esse operador pode ser expresso pela equação 1 a seguir:

$$
\left(\delta^{B}(f)\right)(x)=\max _{b \in B} f(x+b)
$$

Contrariamente, o operador de erosão $\varepsilon$ é o valor mínimo de $f$ na janela definida por $S$ centrada em $x$, podendo ser expresso pela equação 2 :

$$
\left(\varepsilon^{B}(f)\right)(x)=\min _{b \in B} f(x+b)
$$

Quando combinados, os operadoers de dilatação e erosão podem representar outros dois operadores amplamente usados: abertura e fechamento. O operador morfológico de abertura $\gamma$ consiste em dilatar uma imagem $f$ por um elemento estruturante $B$ após uma erosão em $f$ pelo mesmo elemento estruturante. O operador de abertura é denotado, matematicamente, pela equação 3 abaixo:

$$
\gamma^{B}=\delta^{B}\left(\varepsilon^{B}(f)\right)
$$

Por outro lado, o operador morfológico de fechamento $\varphi$ consiste em erodir uma imagem $f$ por um elemento estruturante $B$ após dilatar $f$ pelo mesmo elemento estruturante e é expresso por:

$$
\varphi^{B}=\varepsilon^{B}\left(\delta^{B}(f)\right)
$$

O operador de abertura (ou fechamento) simplifica a imagem ao remover componentes claros (ou escuros) que não se encaixam no elemento estruturante [Ze-Feng et al. 2007].

\subsection{Block-Matching 3D}

Introduzida por [Dabov et al. 2007], a técnica de filtragem por BM3D (Block-Matching $3 D$ ), ou correspondência de blocos $3 \mathrm{D}$, pode ser dividida em três etapas: agrupamento, filtragem colaborativa e agregação.

$\mathrm{Na}$ primeira etapa, conhecida como agrupamento (ou block-matching), a imagem é decomposta em fragmentos (ou blocos) 2D que serão agrupados, de acordo com sua similaridade, em uma matriz de dados 3D (grupos) para passarem pelo processo de filtragem colaborativa [Dabov et al. 2007].

Dado um grupo de $n$ fragmentos, a etapa de filtragem produzirá $n$ estimativas. Primeiramente, é aplicada uma transformação linear de dimensão $d+1$ para o grupo de fragmentos em questão. Após, é feito uma redução dos coeficientes da transformação linear (por limiares suaves, rígidos e filtragem de Wiener) para reduzir o ruído. E, por fim, é realizada a transformada linear inversa para produzir as $n$ estimativas todos os fragmentos filtrados [Dabov et al. 2007].

Por fim, a agregação é feita calculando uma estimativa final da imagem, agregando todas as estimativas, obtidas anteriormente, usando uma média ponderada. [Dabov et al. 2007]. 


\section{Método Proposto}

Primeiramente, a imagem de entrada $f_{1}$, ilustrada pela Figura 1, é pré-processada e convertida em níveis de tons de cinza, denominada $f_{2}$. Além de preservar o contraste ao máximo da imagem original, converter para tons de cinza facilita na distinção de características e no funcionamento dos operadores morfológicos utilizados neste método.

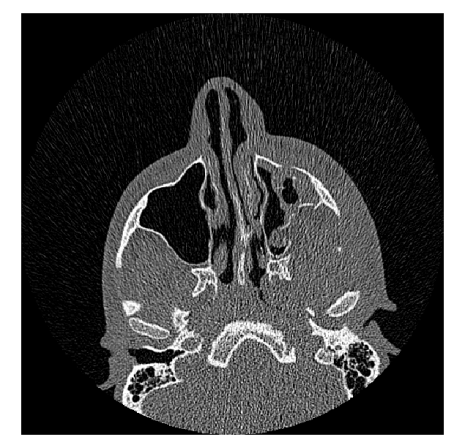

Figura 1. Imagem ruidosa de entrada $f_{1}$.

Em seguida, a fim de reduzir o ruído presente em $f_{2}$, é aplicado o operador morfológico de abertura em $f_{2}$, resultando em $f_{3}$. Como mencionado na Subseção 2.1, os operadores morfológicos extraem informações de uma imagem através de um elemento estruturante. Diante disso, após a realização de diversos experimentos, foi concluído que o elemento estruturante com formato de diamante e tamnho 2 teve melhores resultados, tanto visuais quanto quantitativos. Esse passo pode ser expresso como

$$
f_{3}^{B}=\delta^{B}\left(\varepsilon^{B}\left(f_{2}\right)\right)
$$

onde B é o elemento estruturante. É possível observar que, na Figura 2 (b), a imagem $f_{3}$ teve algumas estruturas e detalhes degradados.

Para realçar detalhes e estruturas importantes que não foram prejudicadas, utilizamos o algoritmo de realce CLAHE (Contrast Limited Adaptive Histogram Equalization) [Pisano et al. 1998]. A técnica divide a imagem em regiões de mesmo tamanho agrupadas em três diferentes grupos de regiões: regiões do canto da imagem, regiões da borda e regiões internas [Reza 2004]. O histograma de cada região é calculado e, por fim, tais regiões são combinadas através de interpolação bilateral, formando a imagem resultante, denominada neste caso de $f_{4}$ e ilustrada na Figura 2 (c).

Agora, para recuperar os detalhes e estruturas degradadas pela abertura morfológica, aplicamos uma reconstrução morfológica por dilatação à imagem realçada $f_{4}$. A reconstrução por dilatação pode ser compreendida como repetidas dilatações de uma imagem, denominada imagem marcadora, até que o contorno dessa imagem seja estabilize sob uma imagem máscara. De acordo com [Soille 2013], a reconstrução por dilatação $R^{\delta}$ de uma marcadora, no nosso caso $f_{4}$, sob a imagem máscara, $f_{2}$ pode ser descrita como sendo

$$
f_{5}=R_{f_{2}}^{\delta}\left(f_{4}\right)=\delta_{f_{2}}^{i}\left(f_{4}\right)
$$

onde $f_{5}$ é a imagem reconstruída, $i$ é tal que $\delta_{f_{2}}^{i}\left(f_{4}\right)=\delta_{f_{2}}^{i+1}\left(f_{4}\right)$. O resultado desse processo é ilustrado através da Figura 2 (d). 
O último passo do método proposto é aplicar o filtro BM3D (Block-Matching 3D), proposto por [Dabov et al. 2007], na imagem resultante do passo anterior, $f_{5}$, para reduzir o ruído remanescente na estrutura circular cinza da imagem. Essa técnica reduz ruido através do processamento de blocos similares dentro de uma imagem. $\mathrm{O}$ algoritmo agrupa blocos de fragmentos 2D da imagem que são similares em uma estrutura de dados 3D (grupos) e realiza uma filtragem colaborativa em tais grupos: em um primeiro momento, através de uma transformação 3D com threshold rígido e, após, através de um filtro de Wiener colaborativo. Por fim, a imagem é reconstruída através de uma agregação por média ponderada. Como resultado do último passo da nossa metodologia proposta, a imagem reconstruída, ou filtrada, é denominada $f_{6}$ e ilustrada pela Figura 2 (e).

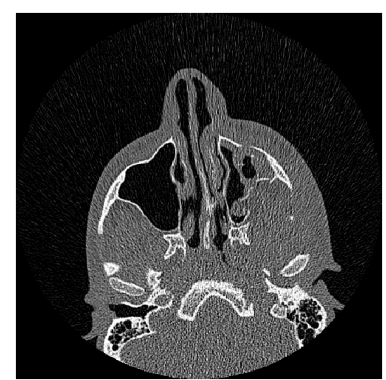

(a)

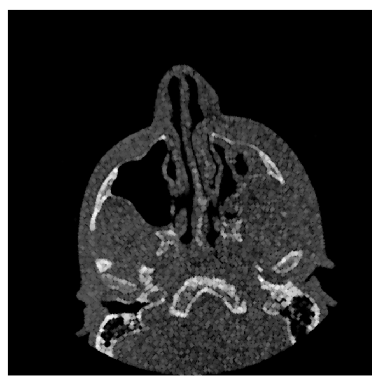

(b)

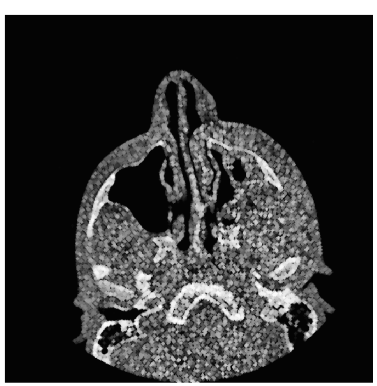

(c)

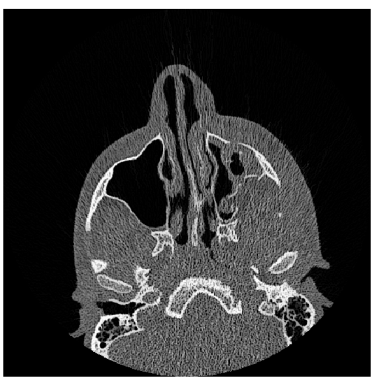

(d)

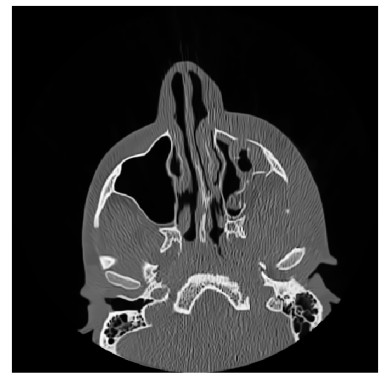

(e)

Figura 2. Exemplo de imagem processada pelo método proposto. (a) Imagem ruidosa em tons de cinza $f_{2}$. (b) Imagem $f_{3}$, resultante do operador de abertura. (c) Imagem $f_{4}$, processada pelo CLAHE. (d) Imagem $f_{5}$ reconstruída. (e) Imagem $f_{6}$ resultante do método proposto, após ser processada pelo BM3D.

O fluxograma a seguir ilustra, de forma resumida e objetiva, o funcionamento e os passos do método proposto para redução de ruído em imagens dentais de tomografia computadorizada de baixa radiação.

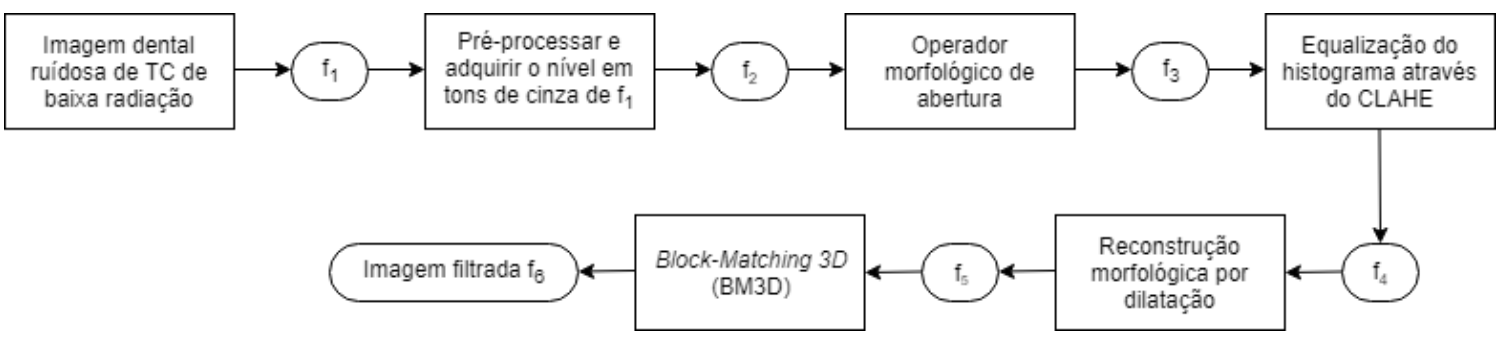

Figura 3. Fluxograma do método proposto. 
A próxima seção apresentará os experimentos realizados e resultados obtidos pelo método proposto em comparação a diversos métodos testados, sendo validados pelas métricas PSNR (Peak Signal-to-Noise Ratio), SSIM (Structural Similarity Index) e MSE (Mean-Squared Error).

\section{Resultados Experimentais}

Para validar o desempenho em reduzir ruído e preservar estruturas do método proposto, experimentos foram realizados em um conjunto de 991 imagens dentais de exames de TC de baixa radiação, retiradas de um dataset local privado anônimo, através da ferramenta MATLAB R2015a. O desempenho da nossa proposta foi comparada com diversos filtros existentes, tais como filtro da mediana, filtro de Wiener, filtro Gaussiano, filtro da média, filtro guiado, filtragem bilateral, filtro bitônico, BM3D, Visu-Shrink, AWMF (Average Weighted Median Filter), difusão anisotrópica e NLM (Non-Local Means). A validação dos resultados foi feita utilizando as métricas quantitativas PSNR, SSIM e MSE que verificam a qualidade da imagem em termos de ruído reduzido e estruturas preservadas.

A métrica PSNR (Peak Signal-to-Noise Ratio) descreve, quantitativamente, a qualidade de uma imagem reconstruída $g$ após processada por algum filtro em relação à imagem original $f$. Matematicamente, é expressa por

$$
\operatorname{PSNR}(f, g)=10 \log _{10}\left(\frac{255^{2}}{M S E(f, g)}\right)
$$

onde MSE é o erro quadrático médio. Quanto maior o PSNR de uma imagem, melhor é a qualidade da mesma.

A métrica MSE mede a diferença quadrática média entre duas imagens $f$ e $g$ através da Equação 8 a seguir:

$$
M S E(f, g)=\frac{1}{M N} \sum_{i=1}^{M} \sum_{j=1}^{N}\left(f_{i j}-g_{i j}\right)^{2}
$$

Quanto menor o valor do MSE entre duas imagens, menor é a diferença entre elas.

Outra métrica importante e utilizada neste estudo é a SSIM (Structural Similarity Index). É usada para medir a similaridade estrutural entre duas imagens $f$ (original) e $g$ (reconstruida). Esta métrica é projetada modelando qualquer distorção da imagem como uma combinação de três importantes fatores que são: perda de correlação, distorção de luminância e distorção de contraste [Hore and Ziou 2010]. Matematicamente, é denotada por:

$$
S S I M(f, g)=l(f, g) c(f, g) s(f, g)
$$

onde

$$
\begin{gathered}
l(f, g)=\frac{2 \mu_{f} \mu_{g}+C_{1}}{\mu_{f}^{2}+\mu_{g}^{2}+C_{1}} \\
c(f, g)=\frac{2 \sigma_{f} \sigma_{g}+C_{2}}{\sigma_{f}^{2}+\sigma_{g}^{2}+C_{2}} \\
s(f, g)=\frac{\sigma_{f g}+C_{3}}{\sigma_{f} \sigma_{g}+C_{3}}
\end{gathered}
$$


tal que $C_{1}=\left(k_{1} L\right)^{2}, C_{2}=\left(k_{2} L\right)^{2}, C_{3}=\frac{C_{2}}{2}, k_{1}=0.01, k_{2}=0.02$ e $L$ é o intervalo dinâmico dos valores dos pixels. O termo $l(f, g)$ refere-se à comparação de luminância onde $\mu$ é a média entre $f$ e $g ; c(f, g)$ é a função de contraste onde $\sigma$ é a variância entre $f$ e $g$ e, por fim, $s(f, g)$ é a função de comparação estrutural. Quando o valor do SSIM tende a 1 , significa que as estruturas foram preservadas e estão de acordo com a da imagem original.

A performance de cada filtro testado e do método proposto em termos de PSNR, medidos em decibéis, é ilustrada pelo gráfico na Figura 4 abaixo.

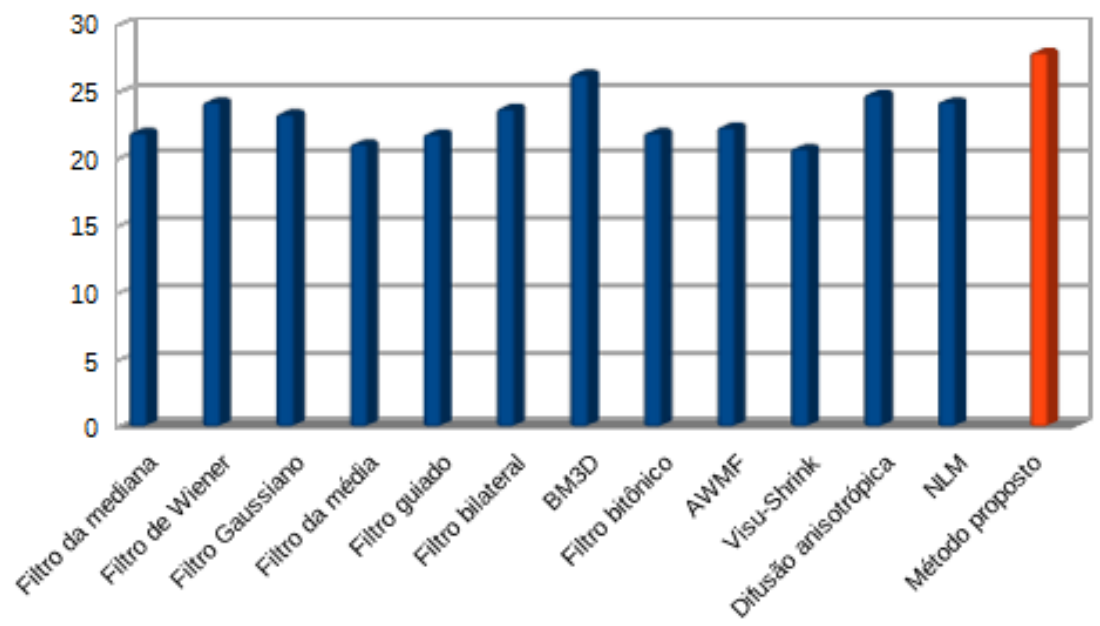

Figura 4. Valores médios de PSNR de cada filtro testado.

De acordo com os resultados médios acima, o nosso método proposto obteve uma performance superior a todos os filtros comparados, com um PSNR médio de $28.09 \mathrm{~dB}$, $6.92 \%$ mais eficiente do que o filtro BM3D. Em termos de similaridade estrutural, nossa proposta também mostrou mais eficiência em preservar detalhes e estruturas das imagens. O valor médio de SSIM obtido foi de $0.71,9.23 \%$ superior que o obtido pelo filtro BM3D, com 0.65 .

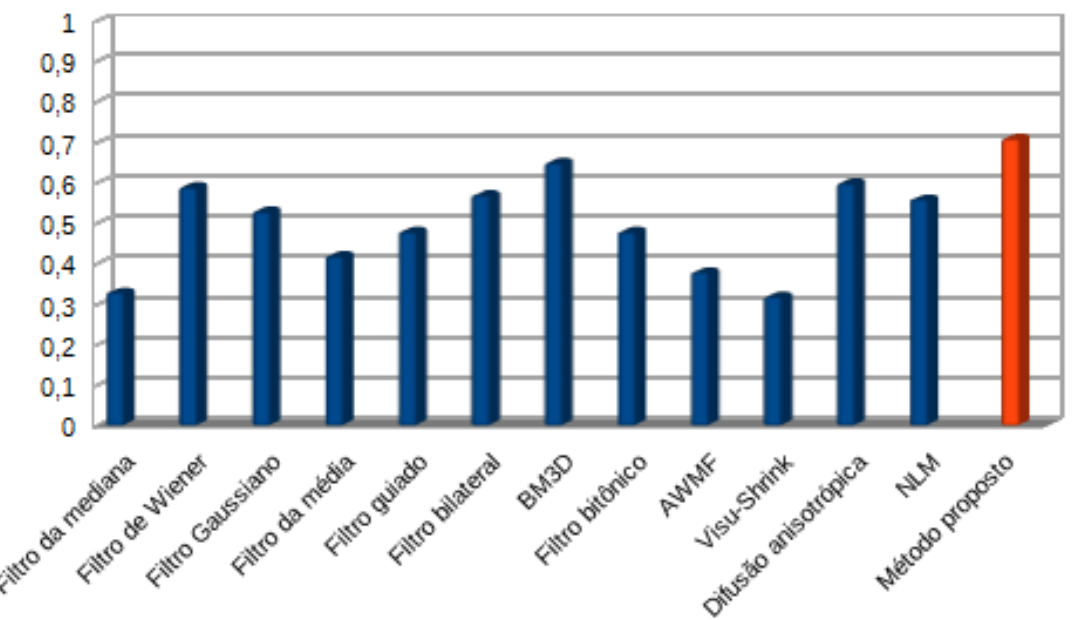

Figura 5. Valores médios de SSIM de cada filtro testado. 
Além disso, nosso método proposto produziu um valor médio de 181.22 na métrica MSE, 8.91\% melhor que o BM3D. Em outras palavras, quanto menor o valor obtido nessa métrica, menor é a perda de informação.

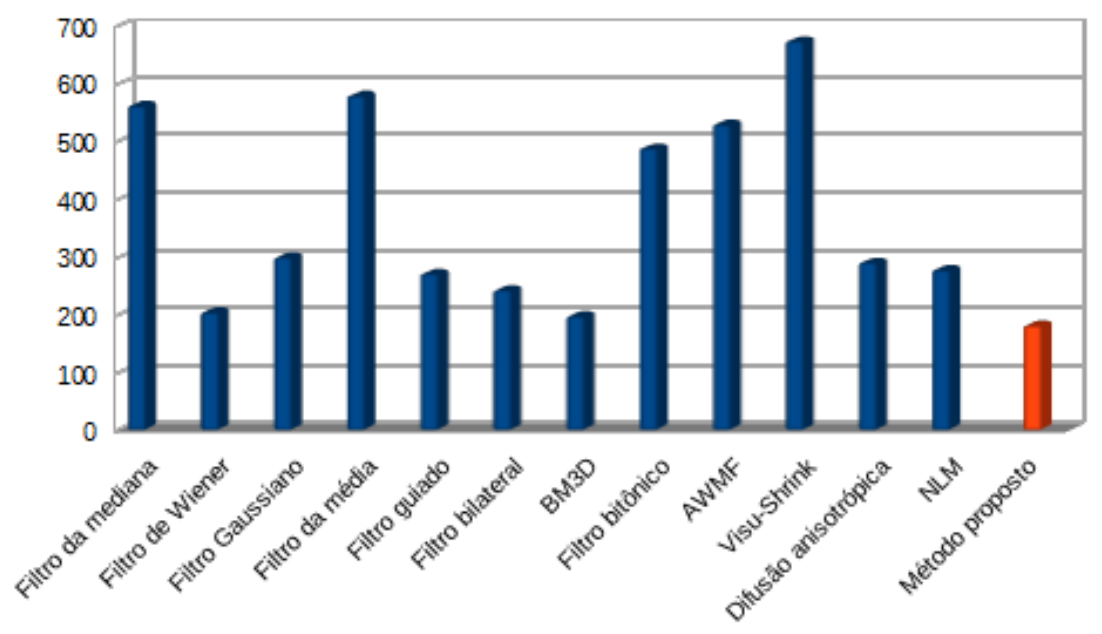

Figura 6. Valores médios de MSE de cada filtro testado.

\section{Análise e Discussão dos Resultados}

Nesta seção é apresentada uma análise mais detalhada sobre os resultados obtidos, em termos quantitativos e visuais, de cada filtro analisado. A Tabela 1 apresenta os valores médios de PSNR, SSIM e MSE obtidos por cada filtro testado, levando em consideração seus melhores parâmetros de configuração.

Tabela 1. Performance de cada filtro analisado.

\begin{tabular}{|c|c|c|c|}
\hline Filters & PSNR & SSIM & MSE \\
\hline Filtro da mediana & 21.32 & 0.33 & 561.95 \\
Filtro de Wiener & 24.76 & 0.58 & 204.31 \\
Filtro Gaussiano & 23.32 & 0.53 & 299.64 \\
Filtro da média & 21.08 & 0.42 & 579.03 \\
Filtro guiado & 21.83 & 0.48 & 271.27 \\
Filtro bilateral & 23.72 & 0.57 & 242.76 \\
BM3D & 26.27 & 0.65 & 197.38 \\
Filtro bitônico & 21.91 & 0.48 & 488.18 \\
AWMF & 22.33 & 0.38 & 529.16 \\
Visu-Shrink & 20.74 & 0.32 & 673.65 \\
Difusão anisotrópica & 24.77 & 0.6 & 288.61 \\
NLM & 24.31 & 0.59 & 277.5 \\
Proposto & $\mathbf{2 8 . 0 9}$ & $\mathbf{0 . 7 1}$ & $\mathbf{1 8 1 . 2 2}$ \\
\hline
\end{tabular}

Os resultados visuais de cada filtro são ilustrados pela Figura 7. A imagem de entrada a ser processada é mostrada pela Figura 7 (a). Na Figura 7 (b) temos a imagem processada pelo filtro da mediana, onde o ruído foi reduzido, entretanto, algumas pequenas estruturas e detalhes foram borrados, visto pelo SSIM médio de 0.33. A Figura 7 
(c) mostra o resultado obtido após uma filtragem de Wiener. Visualmente analisando, ainda é possível observar algum ruído presente na imagem, entretanto, preservou melhor os detalhes do que o filtro da mediana, pois teve um maior SSIM. O mesmo não pode ser observado na Figura 7 (d), processada pelo filtro Gaussiano, pois teve bordas e detalhes severamente borrados, em relação ao filtro de Wiener.

Nas imagens ilustradas pela Figura 7 (e) e Figura 7 (f), processadas pelo filtro da média e pelo filtro guiado, respectivamente, é possível analisar e concluir que a performance em termos visuais foi praticamente a mesma, deixando uma sensação de borramento e fraca suavização de ruído, além de apresentar resultados similares nas métricas quantitativas. A imagem processada pelo filtro bilateral, ilustrada pela Figura 7 (g), teve suas bordas e detalhes preservados de forma semelhante ao filtro de Wiener, provado pelos valores médios de similaridade estrutural próximos. Entretanto, o ruído presente não foi reduzido como desejado. Dentre os filtros analisados, o que apresentou melhor resultados foi o BM3D. Na Figura 7 (h), podemos verificar que o ruído presente foi razoavelmente reduzido, com PSNR médio de $26.27 \mathrm{~dB}$, porém, a técnica preservou eficientemente estruturas e detalhes presentes na imagem, com SSIM médio de 0.65. Além disso, o MSE médio obtido prova que o filtro não produz perda de informação.

A imagem processada pelo filtro bitônico, ilustrada pela Figura 7 (i), teve suas estruturas e detalhes preservados de forma mais eficiente do que o filtro da mediana, com SSIM médio superior, e teve resultados visuais semelhantes ao do filtro Gaussiano. A filtragem AWMF (Average Weighted Median Filter produziu resultados visuais semelhantes ao filtro da mediana, ilustrados pela Figura 7 (j). A diferença encontrada foi que a imagem filtrada pelo filtro AWMF borrou pequenas estruturas de forma mais leve do que o filtro da mediana. A Figura 7 (k), filtrada pela técnica Visu-Shrink, mostra que a imagem foi totalmente degradada, onde artefatos indesejáveis foram gerados e algumas estruturas foram perdidas ou borradas, provando porque os resultados médios para todas as métricas foram os piores. Através da filtragem por difusão anisotrópica, podemos analisar que, tanto pelos valores da Tabela 1 quanto pela Figura 7 (1), estruturas foram preservadas e o ruído foi suavizado. Contudo, é possível verificar a existência de pixels cinzas nas partes escuras da imagem e pixels pretos na estrutura circular cinza. O último filtro analisado foi o Non-Local Means (NLM) e seu resultado é mostrado através da Figura 7 (m). Os resultados quantitativos e visuais são semelhantes ao filtro de Wiener, ou seja, suavizou uma quantidade significativa de ruído enquanto estruturas e detalhes foram preservados.

O resultado da imagem processada pelo método proposto pode ser analisada através da Figura 7 (n). Nossa proposta teve uma performance superior em todas as métricas de qualidade de imagem, assim como um resultado eficiente em aspectos visuais. Com um PSNR médio de 28.09dB, é possivel analisar através da imagem mencionada que o método proposto reduziu em larga escala o ruído que se fazia presente na imagem de entrada. Além disso, com um SSIM médio de 0.71, bordas, estruturas e detalhes pequenos foram eficientemente preservados. A perda de informação foi mínima, com uma média de 181.22 na métrica MSE. 


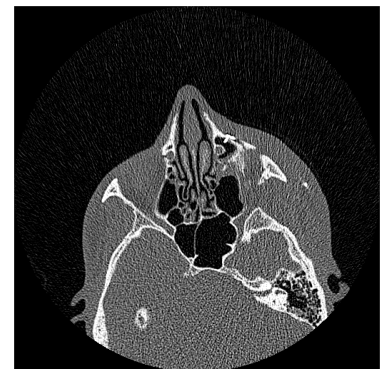

(a)

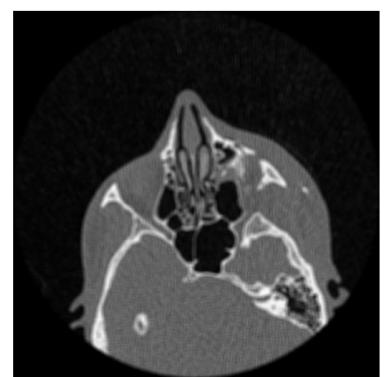

(d)

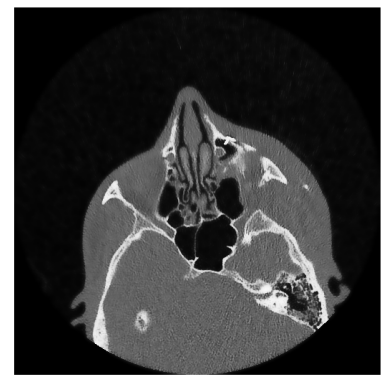

(g)

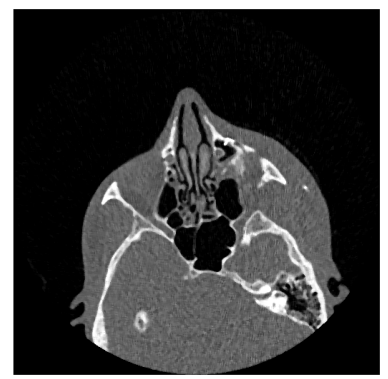

(j)

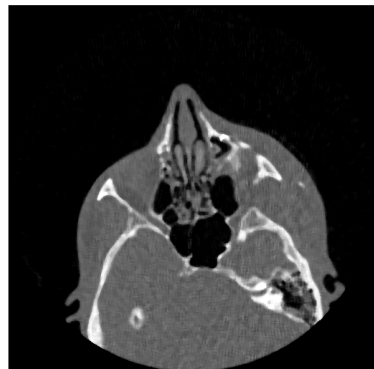

(b)

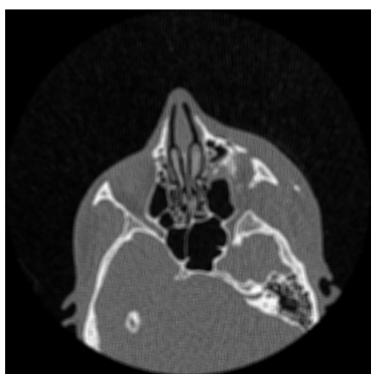

(e)

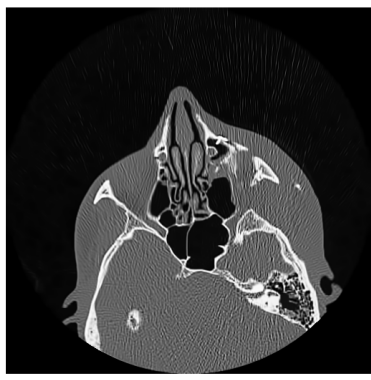

(h)

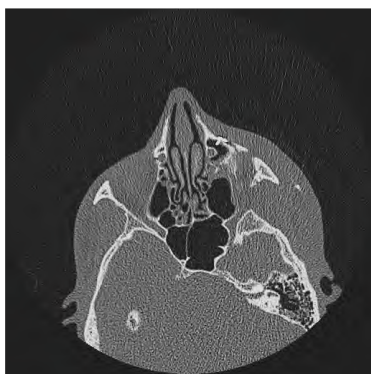

$(\mathrm{k})$

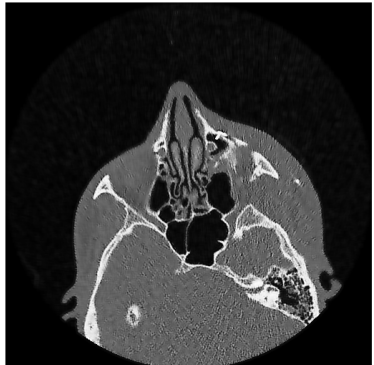

(c)

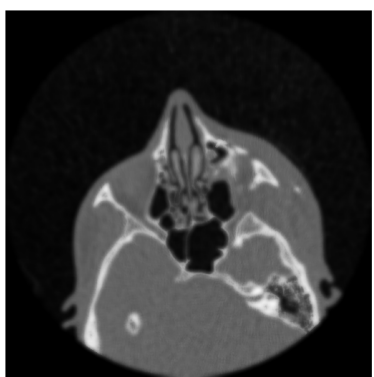

(f)

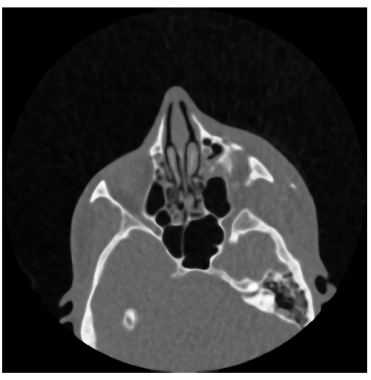

(i)

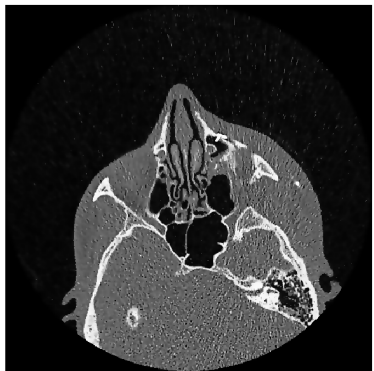

(1)

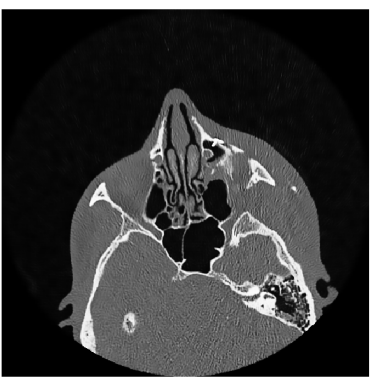

(m)

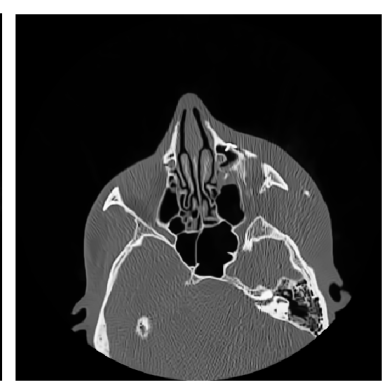

(n)

Figura 7. (a) Imagem ruidosa. (b) Filtro da mediana. (c) Filtro de Wiener. (d) Filtro Gaussiano. (e) Filtro da média. (f) Filtro guiado. (g) Filtro bilateral. (h) BM3D. (i) Filtro bitônico. (j) AWMF. (k) Visu-Shrink. (I) Difusão anisotrópica. (m) NLM. (n) Método proposto. 


\section{Conclusão}

Neste artigo, apresentamos um método baseado em operadores da morfologia matemática em conjunto com filtragem BM3D para reduzir ruído e preservar detalhes em imagens dentais de tomografia computadorizada de baixa radiação. Diversos experimentos foram conduzidos em um conjunto de 991 imagens dentais de tomografia computadorizada de baixa radiação com o intuito de verificar e validar a eficiência do método proposto. Os resultados obtidos indicaram que o método proposto obteve uma performance superior em relação aos filtros comparados em todas as métricas utilizadas para validação. Nossa proposta obteve PSNR médio de 28.09dB, SSIM médio de 0.71 e MSE médio de 181.22. Em comparação com o filtro que obteve os melhores resultados médios dentre os analisados, nossa proposta foi $6.92 \%$ superior na métrica PSNR, 9.23\% na SSIM e $8.91 \%$ na MSE. Além dos bons resultados quantitativos, a performance visual da nossa proposta foi nitidamente superior às técnicas testadas, reduzindo ruído e preservando as estruturas das imagens.

\section{Agradecimentos}

O presente trabalho foi realizado com apoio da Coordenação de Aperfeiçoamento de Pessoal de Nível Superior - Brasil (CAPES) - Código de Financiamento 001.

\section{Referências}

Bergholm, F. (1987). Edge focusing. IEEE Transactions on Pattern Analysis and Machine Intelligence, (6):726-741.

Buades, A., Coll, B., and Morel, J.-M. (2005). A review of image denoising algorithms, with a new one. Multiscale Modeling \& Simulation, 4(2):490-530.

Dabov, K., Foi, A., Katkovnik, V., and Egiazarian, K. (2007). Image denoising by sparse 3-d transform-domain collaborative filtering. IEEE Transactions on image processing, 16(8):2080-2095.

Diwakar, M. and Kumar, M. (2018). A review on ct image noise and its denoising. Biomedical Signal Processing and Control, 42:73-88.

Donoho, D. L. and Johnstone, I. M. (1995). Adapting to unknown smoothness via wavelet shrinkage. Journal of the american statistical association, 90(432):1200-1224.

El Hassani, A. and Majda, A. (2016). Efficient image denoising method based on mathematical morphology reconstruction and the non-local means filter for the mri of the head. In Information Science and Technology (CiSt), 2016 4th IEEE International Colloquium on, pages 422-427. IEEE.

Feng, J., Ding, M., and Zhang, X. (2014). Decision-based adaptive morphological filter for fixed-value impulse noise removal. Optik-International Journal for Light and Electron Optics, 125(16):4288-4294.

He, K., Sun, J., and Tang, X. (2013). Guided image filtering. IEEE transactions on pattern analysis \& machine intelligence, (6):1397-1409.

Hore, A. and Ziou, D. (2010). Image quality metrics: Psnr vs. ssim. In Pattern recognition (icpr), 2010 20th international conference on, pages 2366-2369. IEEE. 
Li, Z., Yu, L., Trzasko, J. D., Lake, D. S., Blezek, D. J., Fletcher, J. G., McCollough, C. H., and Manduca, A. (2014). Adaptive nonlocal means filtering based on local noise level for ct denoising. Medical physics, 41(1).

Loupas, T., McDicken, W., and Allan, P. L. (1989). An adaptive weighted median filter for speckle suppression in medical ultrasonic images. IEEE transactions on Circuits and Systems, 36(1):129-135.

Marques Filho, O. and Neto, H. V. (1999). Processamento digital de imagens. Brasport.

Pisano, E. D., Zong, S., Hemminger, B. M., DeLuca, M., Johnston, R. E., Muller, K., Braeuning, M. P., and Pizer, S. M. (1998). Contrast limited adaptive histogram equalization image processing to improve the detection of simulated spiculations in dense mammograms. Journal of Digital imaging, 11(4):193.

Pitas, I. and Venetsanopoulos, A. N. (1992). Order statistics in digital image processing. Proceedings of the IEEE, 80(12):1893-1921.

Reza, A. M. (2004). Realization of the contrast limited adaptive histogram equalization (clahe) for real-time image enhancement. Journal of VLSI signal processing systems for signal, image and video technology, 38(1):35-44.

Soille, P. (2013). Morphological image analysis: principles and applications. Springer Science \& Business Media.

Tomasi, C. and Manduchi, R. (1998). Bilateral filtering for gray and color images. In Computer Vision, 1998. Sixth International Conference on, pages 839-846. IEEE.

Treece, G. (2016). The bitonic filter: linear filtering in an edge-preserving morphological framework. IEEE Transactions on Image Processing, 25(11):5199-5211.

Ze-Feng, D., Zhou-Ping, Y., and You-Lun, X. (2007). High probability impulse noiseremoving algorithm based on mathematical morphology. IEEE signal processing Letters, 14(1):31-34. 